\title{
Recurrent Spontaneous Colonic Perforation in a 10- years-old Child
}

\author{
Wissem Triki ${ }^{1}$ • Mohamed Amine Lahmidi ${ }^{1}$. Oussema Baraket ${ }^{1}$ - Ahmed Itaim ${ }^{1}$. Sonia Ben Hamida ${ }^{2}$. \\ Abdelmajid Bacar ${ }^{1}$. Sami Bouchoucha ${ }^{1}$
}

Received: 12 December 2018 / Accepted: 4 February 2019 / Published online: 13 February 2019

(C) Dr. K C Chaudhuri Foundation 2019

To the Editor: A 10- y- old infant with no medical history was operated for acute appendicitis. The surgical observation found a gangrened appendix and an appendectomy was performed. Ten days post-operatively, the patient was readmitted for acute peritonitis. A CT-scan showed an abscess adjacent to the sigmoid colon measuring $54 \times 39 \mathrm{~mm}$. Paraoperatively, a sigmoid perforation was found on the anti mesenteric edge. A colonic resection was performed removing the perforation with an end- to- end anastomosis. No lesion that may explain the cause of perforation was found. Thirteen days after the second intervention, the patient was readmitted with symptoms of acute small intestinal obstruction. A CT-scan revealed small intestinal occlusion caused by an adhesion. The surgical observation found an adhesion causing small intestine obstruction and a perforation situated in the middle of the transverse colon on its anti mesenteric edge. The adhesion was dissected and the perforation was removed with an intestinal resection and an end -to- end anastomosis was performed. The histological study of the specimen showed inflammatory phenomena with colonic subserosal necroses and suppurating peritonitis lesions and thrombosed with fibrosis capillaries. The post surgical follow -up was without any complications. The occurrence of spontaneous perforation of colon in older children is extremely rare. The causes of colonic perforation are not clearly established and can be related to many conditions such as infectious diseases, genetic disorders, and vascular conditions. During neonatal period, colonic perforation may be caused by necrotising enterocolitis, Hirschsprung's disease, mechanical obstructions (anorectal malformation,

Wissem Triki

wissem_triki@yahoo.com

1 Department of Surgery, Habib Bougatfa Hospital Bizerte, Bizerte, Tunisia

2 Departement of Gastroenterology, Habib Bougatfa Hospital Bizerte, Bizerte, Tunisia colonic atresia), small left colon syndrome, or rarely, cystic fibrosis [1]. However in older children this pathology has not been extensively studied, and the pathogenesis of this disease has not yet been elucidated. There are several conditions that may cause colonic perforation (CP). It can be related to a genetic disorder such as Ehlers Danlos Syndrome [2]. Moreover, infectious diseases can cause CP such as Typhoid fever. Another cause is idiopathic colonic perforation. It is sporadic and could occur at any age $[3,4]$. The treatment of colonic perforation is essentially surgical and the prognosis depends essentially on the time to diagnosis [5].

Publisher's Note Springer Nature remains neutral with regard to jurisdictional claims in published maps and institutional affiliations.

\section{References}

1. Siddiqui MM, Burge DM. Neonatal spontaneous colonic perforation due to cystic fibrosis. Pediatr Surg Int. 2008;24:863-4.

2. Byers PH. Ehlers-Danlos syndrome: recent advances and current understanding of the clinical and genetic heterogeneity. J Invest Dermatol. 1994;103:47S-52S.

3. Haddad R, Bursle G, Piper B. Stercoral perforation of the sigmoid colon. ANZ J Surg. 2005;75:244-6.

4. Zamir O, Goldberg M, Udassin R, Peleg O, Nissan S, Eyal F. Idiopathic gastrointestinal perforation in the neonate. J Pediatr Surg. 1988;23:335-7.

5. Xu B, Shao WD, Shen HW, Wang QB. Emergence treatment of idiopathic rupture of sigmoid colon. Zhonghua Jizhen Yixue Zazhi. 2004;13:564-6. 